\title{
INFRAFRONTIER quality principles in systemic phenotyping
}

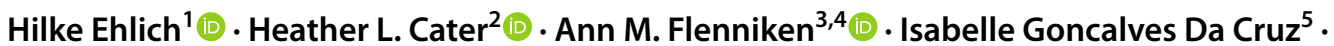

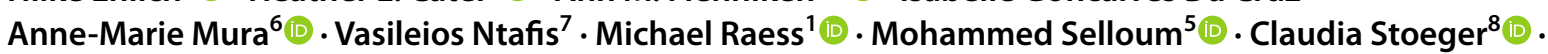

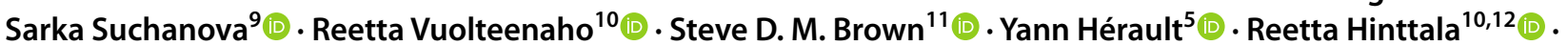

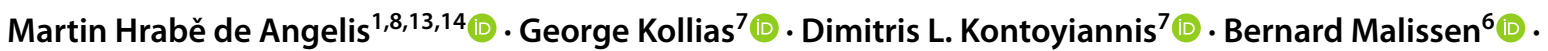

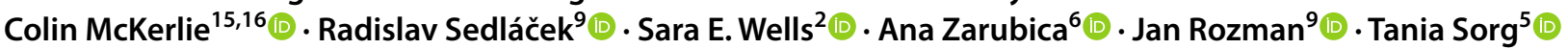

Received: 4 May 2021 / Accepted: 30 June 2021 / Published online: 30 July 2021

(C) The Author(s) 2021, corrected publication 2021

\begin{abstract}
Improving reproducibility and replicability in preclinical research is a widely discussed and pertinent topic, especially regarding ethical responsibility in animal research. INFRAFRONTIER, the European Research Infrastructure for the generation, phenotyping, archiving, and distribution of model mammalian genomes, is addressing this issue by developing internal quality principles for its different service areas, that provides a quality framework for its operational activities. This article introduces the INFRAFRONTIER Quality Principles in Systemic Phenotyping of genetically altered mouse models. A total of $11 \mathrm{key}$ principles are included, ranging from general requirements for compliance with guidelines on animal testing, to the need for well-trained personnel and more specific standards such as the exchange of reference lines. Recently established requirements such as the provision of FAIR (Findable, Accessible, Interoperable, Reusable) data are also addressed. For each quality principle, we have outlined the specific context, requirements, further recommendations, and key references.
\end{abstract}

Jan Rozman

jan.rozman@img.cas.cz

Tania Sorg

tsorg@igbmc.fr

1 INFRAFRONTIER GmbH, Neuherberg, Germany

2 MRC Harwell Institute, Mary Lyon Centre, Harwell Campus, Oxfordshire, UK

3 Lunenfeld-Tanenbaum Research Institute, Sinai Health, Toronto, ON, Canada

4 The Centre for Phenogenomics, Toronto, ON, Canada

5 Universite de Strasbourg, CNRS, INSERM, CELPHEDIA, PHENOMIN, Institut Clinique de La Souris, Illkirch, France

6 Centre d'Immunophénomique (CIPHE), Aix Marseille Université, INSERM, CNRS, CELPHEDIA, PHENOMIN, Marseille, France

7 Biomedical Sciences Research Center "Alexander Fleming", Athens, Greece

8 Helmholtz Zentrum München, Institute of Experimental Genetics, German Mouse Clinic, Neuherberg, Germany
9 Czech Centre for Phenogenomics, Institute of Molecular Genetics of the Czech Academy of Sciences, Vestec, Czech Republic

10 University of Oulu, Biocenter Oulu, Transgenic and Tissue Phenotyping Core Facility, Oulu, Finland

11 MRC Harwell Institute, Mammalian Genetics Unit, Medical Research Council, Harwell Campus, Oxfordshire, UK

12 University of Oulu and Oulu University Hospital, PEDEGO Research Unit and Medical Research Center Oulu, Oulu, Finland

13 German Center for Diabetes Research (DZD), Neuherberg, Germany

14 Technische Universität München, Chair of Experimental Genetics, TUM School of Life Sciences, Freising, Germany

15 The Hospital for Sick Children, Toronto, ON, Canada

16 The Faculty of Medicine, University of Toronto, Toronto, ON, Canada 


\section{Introduction}

Low reproducibility in preclinical research is a widely-debated topic. The consequences of irreproducibility are manifold, the most obvious being reduced efficiency in the development of new treatments, the cost in animal lives, the waste of billions of US\$ per year in the USA alone (Freedman et al. 2017) as well as the loss of trust in science.

Various efforts have been undertaken to increase reproducibility in animal research. Guidelines for experimental design and reporting have been formulated, e.g. PREPARE (Smith et al. 2018) and ARRIVE guidelines (Kilkenny et al. 2010, Percie du Sert 2020a, b), and funding bodies are actively fostering the sharing of data which is open and FAIR (Findable, Accessible, Interoperable and Reusable; Wilkinson et al. 2016) (European Open Science Cloud in the EU, Science Data Commons in the US).

In addition, large research consortia, such as the International Mouse Phenotyping Consortium (IMPC), which aim to phenotype knockouts for all protein-coding genes in the mouse genome, are driving improvements through standardisation of procedures and sharing of data.

INFRAFRONTIER is the European Research Infrastructure for the generation, phenotyping, archiving and distribution of model mammalian genomes. INFRAFRONTIER provides centralised access to mouse and rat models, data, and scientific platforms and services to study the functional role of the genome in human health and disease. The core services comprise model generation, archiving and distribution of mouse mutant lines by the European Mouse Mutant Archive (EMMA), and systemic phenotyping of mouse mutants in the participating mouse clinics. Systemic phenotyping, as used in the INFRAFRONTIER context, means the systematic analysis of mutant mice through a standardised phenotyping pipeline across a range of biological systems to infer gene function.

The INFRAFRONTIER consortium is currently formed by 29 partners across 14 European countries and Canada, with most of the mouse clinics also being members of the IMPC network. Accordingly, its contribution to enhancing reproducibility in biomedical research is characterised by providing centralised open access to high-quality scientific data, biomedical services and resources, and through the sharing of knowledge. As a large distributed research infrastructure, it is the mission of INFRAFRONTIER to deliver reliable services and reproducible data while advancing animal research in accordance with the 3Rs (Replacement, Reduction, Refinement).

\section{INFRAFRONTIER quality principles in systemic phenotyping}

INFRAFRONTIER relies on the development of internal quality principles to provide a quality framework for its operational activities and to guide continuous improvement in response to the needs of users and stakeholders. Incorporating both existing operational standards and common concepts in Quality Management (QM), these principles define internal key quality standards for each INFRAFRONTIER service. In addition, current developments and insights towards enhanced research reproducibility are addressed. These include providing transparent, FAIR and open data, following the ARRIVE and PREPARE guidelines, and using the most advanced experimental approaches. Since INFRAFRONTIER is a consortium of many research institutions across different countries, the Quality Principles recognise that requirements at each of the national nodes may differ and that different strategies may be chosen for the application of the defined standards.

This article provides a complete overview of the INFRAFRONTIER Quality Principles in Systemic Phenotyping. These principles were established by the INFRAFRONTIER Quality Management Network Group, an internal INFRAFRONTIER group composed of representatives of the INFRAFRONTIER partner institutions, who are dedicated to the exchange of current QM topics. The group identified the following 11 principles as crucial for providing reliable, high-quality phenotyping services:

(1) We strictly comply to national and international legislation on ethics and animal welfare

(2) We promote and apply the 3Rs (Replacement, Reduction, Refinement)

(3) We apply good experimental practice

(4) We apply Standard Operating Procedures

(5) We ensure that our procedures are carried out by competent and well-trained personnel

(6) We apply reference ranges where feasible

(7) We exchange and analyse reference lines or reference samples where feasible

(8) We use appropriate statistical analyses that are fit for purpose

(9) We report metadata

(10) We advise that the data that we provide is FAIR (Findable, Accessible, Interoperable, Reusable)

(11) We maintain and extend the mechanisms (working groups, training, exchange of experience) to constantly improve our data quality (Plan-Do-Check-Act)

In addition, the partners elaborated on context, specific requirements, recommendations, and supporting references sections for each of the 11 principles. 
In this respect, the context sections are meant to "set the scene". They provide information about the underlying background of the principles, for example specific conditions and circumstances which need to be considered. Thus, they point out the relevance of the specific principles for INFRAFRONTIER.

The requirement sections list mandatory points, such as procedures which should be implemented at the nodes to fulfil the needs of the relevant principle.

Additionally, the recommendation section of each principle proposes further procedures and measures which will improve adherence to the principles but are not mandatory.

Finally, the references list relevant regulations and publications, as well as links to webpages which are referred to in the other sections or provide useful information.

\section{Conclusion}

By defining crucial quality criteria, the INFRAFRONTIER quality principles establish the basis for reproducible and reliable research results. They provide the participating partners with a sound framework for their operational activities in systemic phenotyping while at the same time providing leeway for the implementation of and adherence to national and local requirements.

Supplementary Information The online version contains supplementary material available at https://doi.org/10.1007/s00335-021-09892-2.

Acknowledgements We would like to thank all the members of the INFRAFRONTIER consortium and especially Helmut Fuchs and Lluis Montoliu for their valuable feedback. Likewise, we would like to thank Nicole Linklater.

Authors contribution HE, HLC, AMF, IG, AMM, VN, MR, MS, CS, SS, RV, JR, and TS contributed to defining the quality principles and drafting the manuscript. SDMB, YH, RH, MHdA, GK, DK, BM, CM, RS, SEW, and AZ approved the quality principles. All authors read and approved the final manuscript.

Funding This work has been funded by the European Union Research and Innovation programme Horizon 2020 (INFRAFRONTIER2020Grant Agreement Number 730879). Biomedical Sciences Research Center "Alexander Fleming" acknowledges support of this work by the InfrafrontierGR action, co-funded by Greece and the European Union (European Regional Development Fund), under NSRF 20142020, MIS 5002135. CELPHEDIA-PHENOMIN-ICS and CIPHE are supported from French state funds through the "Agence Nationale de la Recherche" under the frame programme Investissements d'Avenir labelled ANR-10-INBS-07 PHENOMIN. The Czech Centre for Phenogenomics is supported by the Czech Academy of Sciences RVO 68378050 and by the project LM2018126 Czech Centre for Phenogenomics provided by Ministry of Education, Youth and Sports of the
Czech Republic. The German Mouse Clinic is supported by the German Federal Ministry of Education and Research (Infrafrontier Grant 01KX1012). The MRC Harwell Institute is supported by the UKRI Medical Research Council. The Centre for Phenogenomics is supported by Ontario Genomics with a Genomics Technology Platforms grant and by the Canadian Foundation for Innovation Major Sciences Initiatives Grant 35534. Biocenter Oulu, Transgenic and Tissue Phenotyping Core Facility, University of Oulu is supported by the Biocenter Finland and the Academy of Finland, project 319405.

\section{Declarations}

Conflict of interest The authors declare that they have no conflict of interest for this article.

Open Access This article is licensed under a Creative Commons Attribution 4.0 International License, which permits use, sharing, adaptation, distribution and reproduction in any medium or format, as long as you give appropriate credit to the original author(s) and the source, provide a link to the Creative Commons licence, and indicate if changes were made. The images or other third party material in this article are included in the article's Creative Commons licence, unless indicated otherwise in a credit line to the material. If material is not included in the article's Creative Commons licence and your intended use is not permitted by statutory regulation or exceeds the permitted use, you will need to obtain permission directly from the copyright holder. To view a copy of this licence, visit http://creativecommons.org/licenses/by/4.0/.

\section{References}

Freedman LP, Venugopalan G and Wisman R. (2017) Reproducibility2020: Progress and priorities. F1000 Research 2017, 6:604 https://doi.org/10.12688/f1000research.11334.1

Kilkenny C, Browne WJ, Cuthill IC, Emerson M, Altman DG (2010) Improving bioscience research reporting: The ARRIVE guidelines for reporting animal research. PLoS Biol 8(6):e1000412. https://doi. org/10.1371/journal.pbio.1000412

Percie du Sert N, Hurst V, Ahluwalia A, Alam S, Avey MT, Baker M et al (2020) The ARRIVE guidelines 2.0: Updated guidelines for reporting animal research. PLoS Biol 18(7):e3000410. https://doi. org/10.1371/journal.pbio.3000410

Percie du Sert N, Ahluwalia A, Alam S, Avey MT, Baker M, Browne WJ et al (2020) Reporting animal research: explanation and elaboration for the ARRIVE guidelines 2.0. PLoS Biol 18(7):e3000411. https:// doi.org/10.1371/journal.pbio.3000411

Smith AJ, Clutton RE, Lilley E, Aa Hansen KE, Brattelid T (2018) PREPARE: guidelines for planning animal research and testing. Lab Anim 52(2):135-141. https://doi.org/10.1177/2F0023677217724 823

Wilkinson M, Dumontier M, Aalbersberg I et al (2016) The FAIR guiding principles for scientific data management and stewardship. Sci Data 3:160018. https://doi.org/10.1038/sdata.2016.18

Publisher's Note Springer Nature remains neutral with regard to jurisdictional claims in published maps and institutional affiliations. 